\title{
A mathematical model of a simple human galvanic skin response based upon its rate topography
}

\author{
DARWIN P. HUNT \\ New Mexico State University, Las Cruces, New Mexico 88008
}

\begin{abstract}
A mathematical description of a three-state (nonresponse, response, and recovery) model of a simple human galvanic skin response is presented. The model is based upon the temporal topography of the rate of change of the skin resistance. The equation implies that the portions of the topography representing the response and recovery states may be usefully portrayed as overlapping Gaussian distributions. Examples of the agreement between observed values and values based upon the equation are provided.
\end{abstract}

A model of a single galvanic skin response (GSR) which is based upon the topography of the first derivative or rate of change $(\Delta R)$ of the skin resistance (R) has been proposed (Hunt, 1975). It was suggested that the $\Delta \mathrm{R}$ exhibits an orderliness which remains concealed if one considers the $R$ alone, and that the general form of the $\Delta R$ topography of a simple single GSR, which is shown in Figure 1, is relatively invariant.

As is shown at the top of Figure 1, the model consists of three states: a nonresponse (nr) state, a response (r) state, and a recovery (c) state. Since the 1975 description, mathematical equations for the three states have been sought to describe more precisely the apparent invariance. The purpose of this paper is to summarize this attempt at quantification and to report the kind of equation which seems most reasonable at present.

\section{METHOD}

Values of $\mathbf{R}$ were obtained from human subjects by converting the analog values of $R$ measured by a modified Fels Dermohmmeter into digital form. The digitized $R$ values were recorded each $200 \mathrm{msec}$ beginning $200 \mathrm{msec}$ after the onset of a stimulus (a 40-dB tone) and continuing for $8 \mathrm{sec}$. The measurements were recorded to the nearest $10 \mathrm{ohms}$. The $\Delta R$ values were obtained by determining the difference between successive $R$ values. The first $\Delta R$ value following the stimulus onset was calculated by subtracting the $R$ value at $400 \mathrm{msec}$ from the $R$ value at $200 \mathrm{msec}$ and is plotted as having occurred at the midway time of $300 \mathrm{msec}$. Each of the $\Delta R$ values is expressed in terms of the ohms change/sec, for example, $-250 \mathrm{ohms} / \mathrm{sec}$ means that the $R$ decreased $50 \mathrm{ohms}$ during a particular $200-\mathrm{msec}$ interval. These $\Delta \mathbf{R}$ values plotted as a function of time from .3 to $8.1 \mathrm{sec}$ provide the rate topographies of interest.

\section{RESULTS AND DISCUSSION}

With regard to the nr state, visual inspection of the

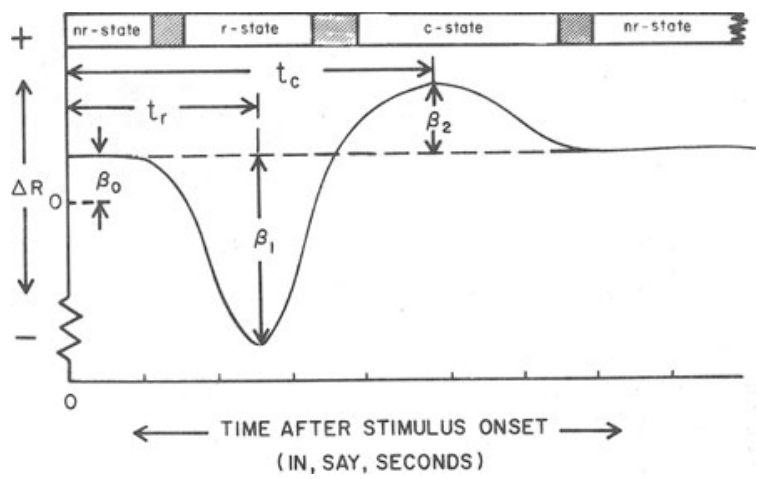

Figure 1. A proposed topography of the first derivative ( $\triangle R$ or rate of change) of the skin resistance in a simple GSR. $\beta_{0}$ is the magnitude of the $\Delta R$ for the nonresponse state; $\beta_{1}$ is the peak $\Delta R$ during the response state; $t_{r}$ is the time of occurrence of $\beta_{1} ; \beta_{2}$ is the peak $\Delta R$ during the recovery state; and $t_{c}$ is the time of occurrence of $\beta_{2}$.

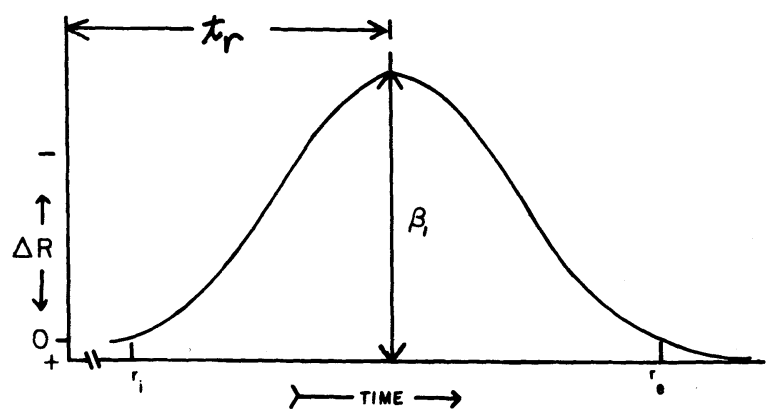

Figure 2. A Gaussian model of the $r$ state where $\beta_{1}$ is the peak $\Delta R$ attained and $t_{r}$ is the time of its occurrence; $r_{i}$ and $r_{e}$ indicate the time of initiation and ending of the $r$ state. 
$\Delta \mathrm{R}$ topographies of many subjects under a variety of conditions suggests that the $\Delta R$ is relatively stable during the $1 \mathrm{sec}$ or so following the onset of a stimuluseven though the $R$ itself may be changing rapidly. Thus, the $\mathrm{nr}$ state may be described simply by saying that the $\Delta R$ is constant $\left(\beta_{0}\right.$ in Figure 1$)$-with some random variation.

Attempts to fit various equations to the $r$ state resulted in (1) a tentative rejection of a parabolic function, a binomial model, and a Poisson model, (2) a tentative assumption that the GSR was always initiated $1.2 \mathrm{sec}$ prior to the peak $\Delta R$ of the $r$ state, (3) the observation that a Gaussian (inverted) model (see Figure 2) fits the data fairly well for the time period extending from the initiation of the response to the peak $\Delta R$ of the $r$ state (see Figure 3), and (4) the observation that the Gaussian model provides a poor fit to

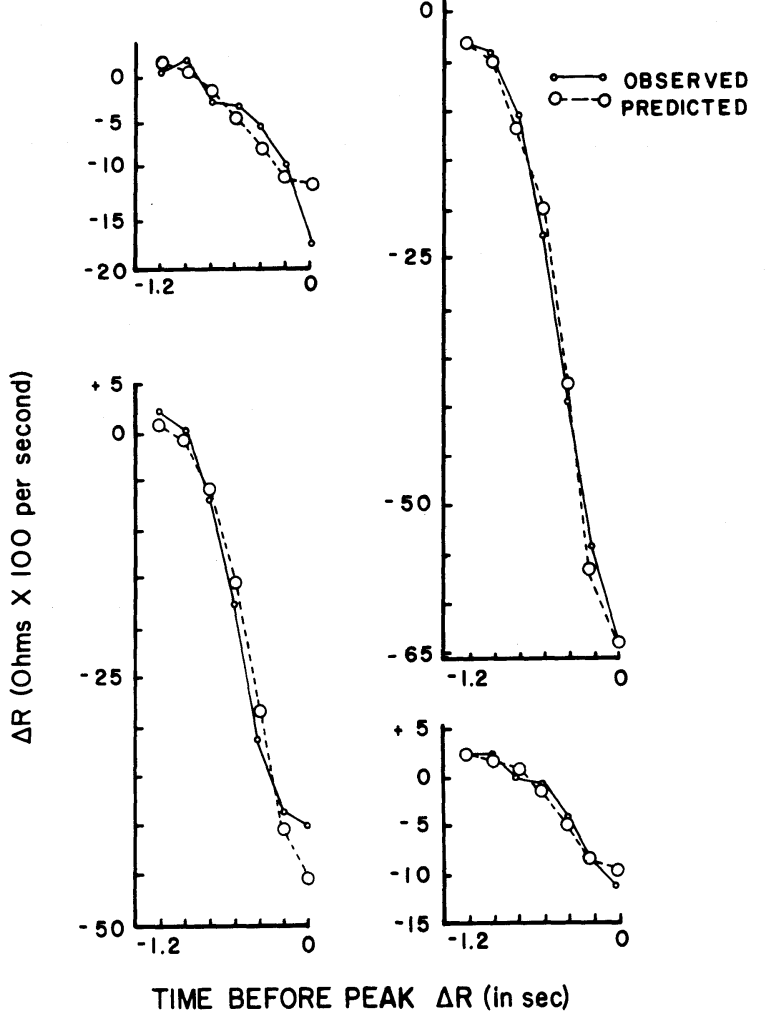

Figure 3. The observed and predicted $\Delta R$ values as a function of time for four different subjects. The predictions are based upon a Gaussian model fitted over the time period from $1.2 \mathrm{sec}$ before the peak $\Delta \mathbf{R}$ to the peak. the data from the peak $\Delta R$ of the $r$ state to the end of the r state.

The apparent asymmetry of the $r$ state suggested that it may be worthwhile to consider the $r$ state and the $c$ state simultaneously. Since the first part of the $r$ state seemed to be Gaussian in form, the notion of overlapping Gaussian distributions for the $r$ state and $\mathrm{c}$ state was explored. This approach produced Equation 1. The meanings of the symbols used in Equation 1 are shown in Figure 1.

$$
\begin{aligned}
\Delta R^{\prime}=f(t)=\beta_{0} & -\frac{\left|\beta_{1}\right|}{e\left[\frac{t-t_{r}}{\left.\left(\frac{t+.4 t_{r}+1.2}{t_{r}}\right) \sigma_{r}\right]^{2}}\right.} \\
& +\frac{\beta_{2}}{e^{\left[\frac{t-t_{c}}{\left(\frac{t+.4 t_{r}+1.2}{t_{r}}\right)} \sigma_{c}\right]^{2}}} .
\end{aligned}
$$

A few examples of the extent of agreement between the values based upon Equation 1 and the actual observations are presented in Figure $4 a, b, c$, and d. These data are single responses of single subjects, and were selected from among 135 students in introductory psychology who served in a previous study of differential GSR conditioning (Hunt, 1975). These subjects were selected for illustration because their $\Delta \mathrm{R}$ values (1) were reasonably stable for the time period from the onset of the stimulus to $1.2 \mathrm{sec}$ later, (2) monotonically attained a peak in the $r$ state once a response was initiated, and (3) monotonically attained a peak in the c state, that is, the $\Delta \mathrm{R}$ smoothly attained a value above the $\Delta \mathbf{R}$ value of the $\mathrm{nr}$ state. These selection criteria were intended to exclude subjects who either were not in a nr state at the time of the stimulus onset and/or made multiple responses.

The linear correlations between the observed $\Delta R$ values and the $\Delta R$ values based upon Equation 1 are $.99, .98, .99$, and .99 for the responses presented in Figure 4a, b, c, and d, respectively. These high correlations are taken as an indication of the possible validity 

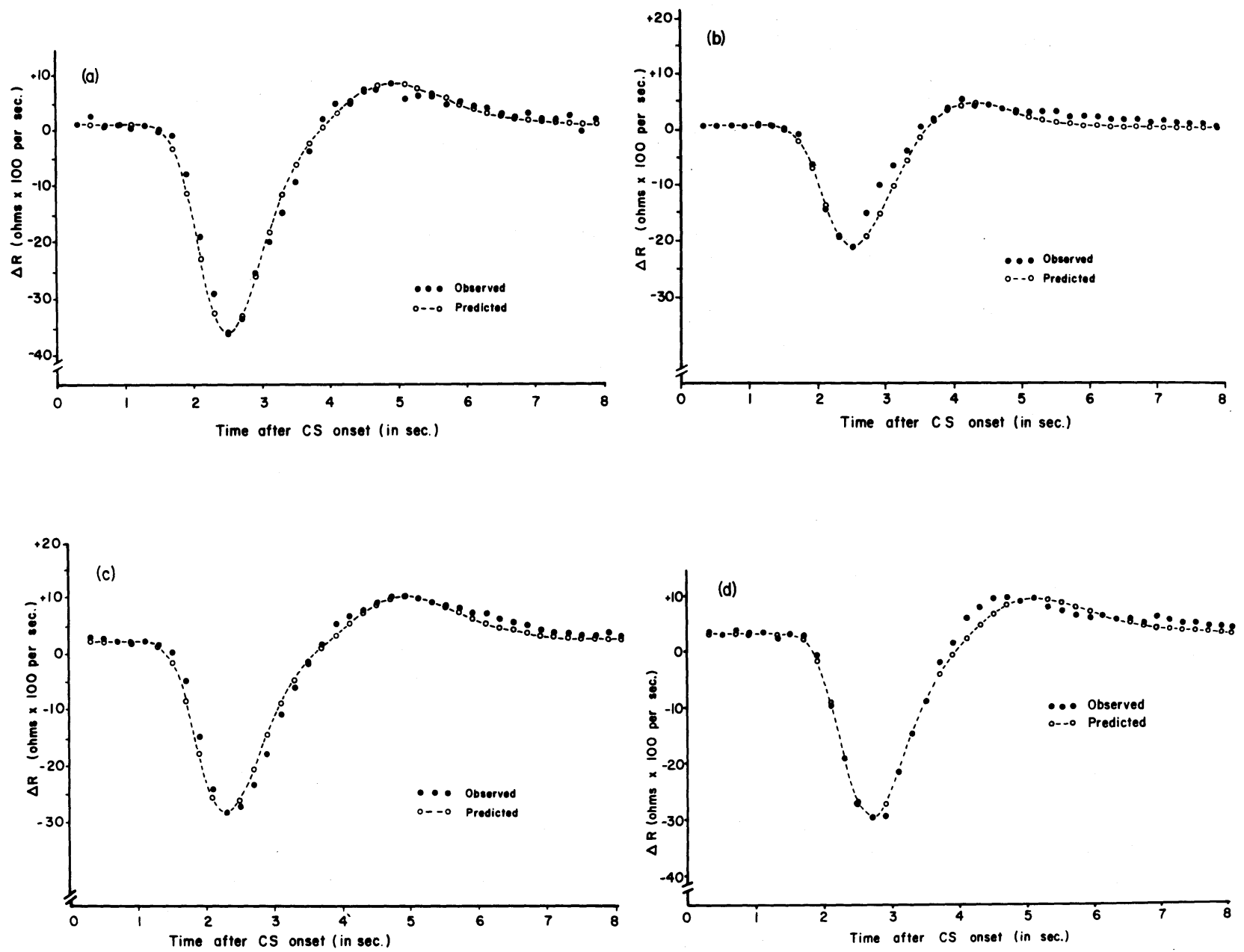

Figure 4. The observed and predicted $\Delta R$ values at $200-\mathrm{msec}$ intervals over a period of time from 300 msec after the onset of a conditioned stimulus to $7.8 \mathrm{sec}$ later. The data are from four different subjects on a test trial which had been preceded by (a) 10 tone-shock pairings with an interstimulus interval of $.5 \mathrm{sec}$, (b) 15 tone-shock pairings with an interstimulus interval of 3.0 sec, (c) 5 tone-shock pairings with an interstimulus interval of $4.0 \mathrm{sec}$, or (d) 10 tone-shock pairings with an interstimulus interval of $4.0 \mathrm{sec}$.

of the general notion of the invariant form of the first derivative of the response and encourages the author to pursue further the approach of overlapping distributions.

This effort began with the goal of developing a model of a single GSR which could serve as a suitable baseline for the evaluation of multiple electrodermal responding. However, the kind of equation presented here also suggests (1) response measures which may be more reliable and more valid, and (2) some avenues to pursue concerning possible processes and/or structures underlying electrodermal responding.

\section{REFERENCE}

Hunt, D. P. Measurements of a single galvanic skin response based upon its rate topography. Journal of General Psychology, 1975, 93, 155-171.

(Received for publication April 4, 1977.) 